\title{
Simulation of checking of mechanical state of the transformer windings by nanosecond impulse
}

\author{
A.S. Semenov, V.A. Lavrinovich, M.T. Pichugina, G.A. Nizkodubov \\ National Research Tomsk Polytechnic University, 634050 Tomsk Russia
}

\begin{abstract}
The detailed electrical model of the transformer windings was developed. The possibility of detecting negligible changes in the geometrical pattern of the windings was investigated by testing of the impulses with nanosecond rising edge.
\end{abstract}

\section{INTRODUCTION}

Monitoring and diagnosis of power transformer windings, voltage class $110 \mathrm{kV}$ and above, has become an urgent problem due to the excess of their service life and the difficulty in replacing expensive equipment by the new one for economic reasons [1]. Therefore, it is of paramount importance to carry out a diagnostic check in order to maintain the required operational reliability of transformers. Diagnostic methods, sensitive to changes in mechanical condition of the windings, are considered to be: short circuit resistance measurement, method of low-voltage pulses (LVP), and method of frequency response analysis [2].

Monitoring and diagnosis by LVP has higher sensitivity than measuring short circuit resistance. A mathematical modeling of a diagnostic/probe signal (signal which passed through a transformer winding) with its follow-up spectral analysis has been carried out for technical implementation of LVP in the form of an automated diagnose system [3].

It is possible to increase sensitivity of the LPV method by nanosecond pulses with a front of $10 \ldots 25 \mathrm{~ns}$ for sensing transformer windings. This is explained by the fact that, even at very small mechanical displacements in the windings, capacity of their individual elements (interturn, intercoil) usually changes significantly. [4].

\section{SiMULATION}

In the circuit simulation program, Micro-Cap 8 a test transformer circuit has been simulated by LVP. A rectangular pulse with duration $\mathrm{t}=400 \mathrm{~ns}$ with a front of about $25 \mathrm{~ns}$ has been formed by a cable generator and fed to the high-voltage winding of the transformer with 120 turns (point "a", Fig. 1). Impulse-response has been removed from the low voltage transformer winding with 20 turns (point "a"). The simulation results obtained by the Micro-Cap program have been compared with the results obtained by a physical model of the transformer (Fig. 1).

Impulse-responses obtained from electric transformer model with defects (defectogram) and bunch graphs obtained from electric transformer model without any defects have been obtained for comparative analysis. Oscillogram-responses have been overlapped. Transients were superimposed on the time axis during overlapping and deviations of defectograms and bunch graphs were determined.

Inter-winding faults, circuit breaks and turns displacements have been considered as defects in lowvoltage (LV) as well as high-voltage (HV) windings.

Figure 2 shows waveforms (a) and simulation results (b) without any defects in the circuit of transformer windings.

The results of simulation tests of transformer short circuits and open windings are shown in Figures 34. They show combined impulses-responses taken at the point ("a", Fig. 1) on a defect-free transformer and the transformer with some defects.

Corresponding author: sayal@sibmail.com

This is an Open Access article distributed under the terms of the Creative Commons Attribution License 4.0, which permits unrestricted use, distribution, and reproduction in any medium, provided the original work is properly cited. 


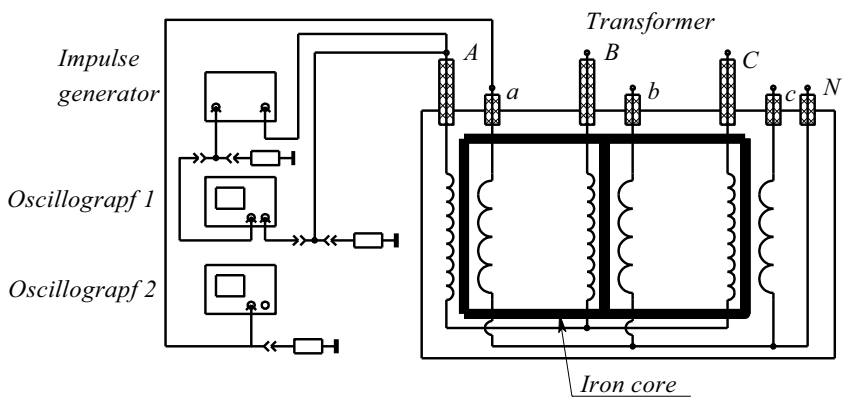

Figure 1. Experimental setup scheme
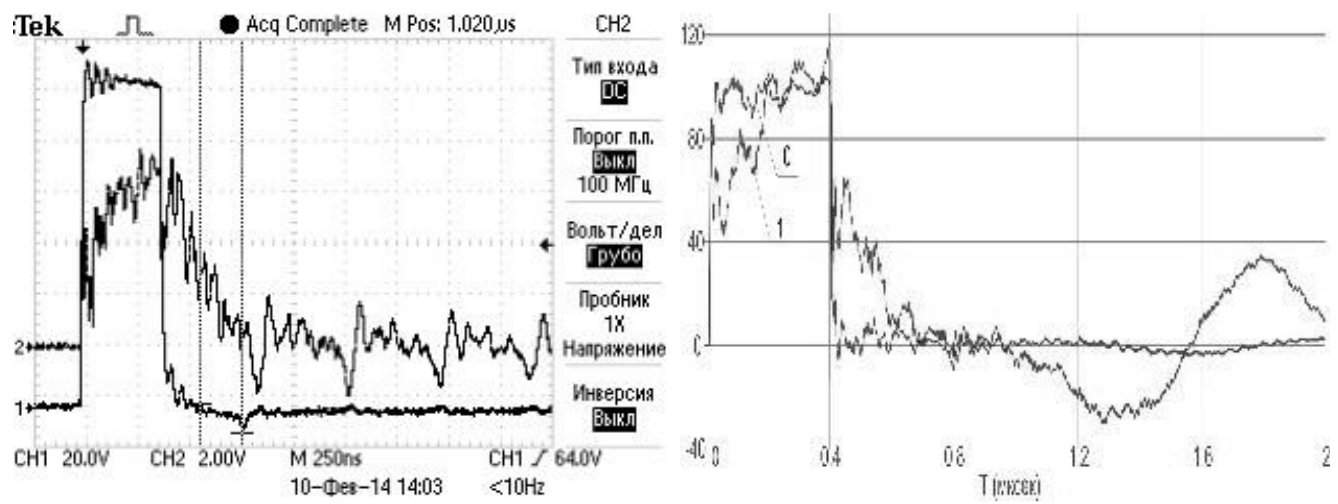

Figure 2. NS pulse waveform combination and its response: a) physical experiment, b) modeling of pulse generator (Curve 0 ) and its response (curve 1) in the defect-free scheme

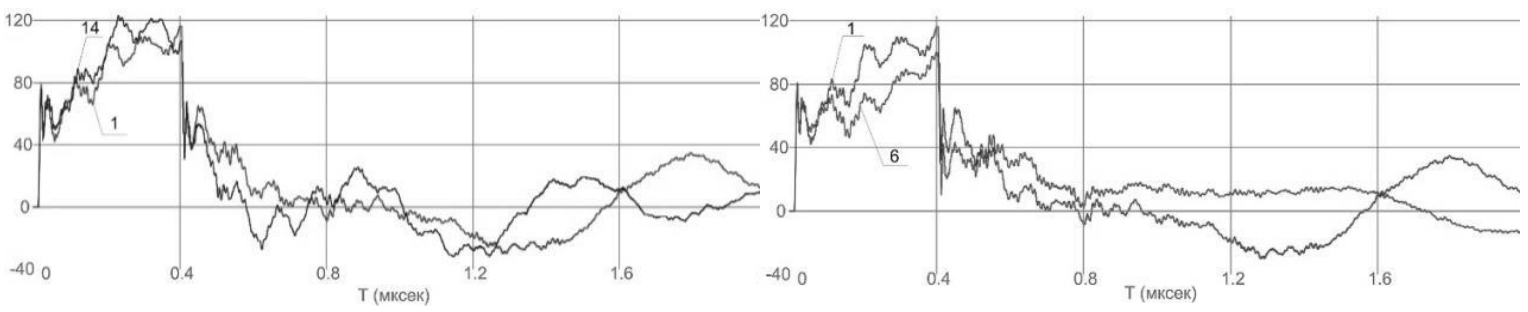

Figure 3. Combined impulses-responses: curve 1 without any defects, curve $14-24$ shorted turns in a HV winding

Figure 4. Combined impulses-responses: curve 1 without any defects, curve 6 - break in a LV winding

Results of simulation transformer tests with displaced winding turns are shown in Figures 5-6. They show combinations of impulses-responses taken at the point ("a", Fig. 1) on a defect-free transformer and the transformer with displaced turns. 


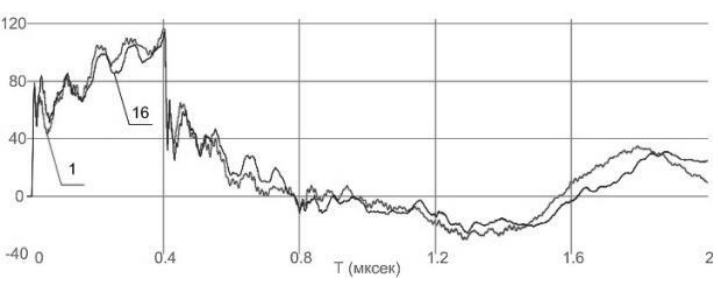

Figure 5. Combined impulses-responses: curve 1 without any defects, curve $16-24$ displaced turns in a HV winding

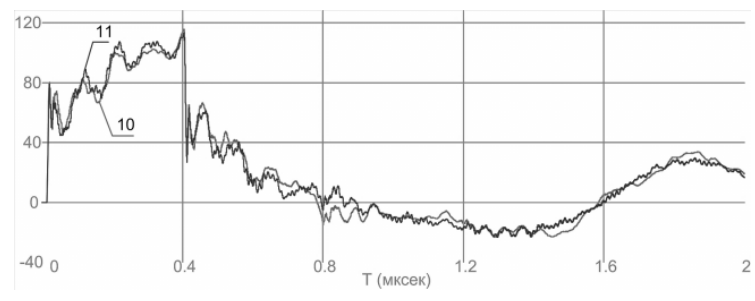

Figure 6. Combined impulses-responses: curve 10 dispaced loops at the beginning of in $\mathrm{HV}$ windings, curve 11 - the same.

In order to test front duration of the probe pulse we carried out a simulation transformer test with the same defects but with front duration of the probe pulse up to $400 \mathrm{~ns}$. The simulation results are shown in Figures 7-8.

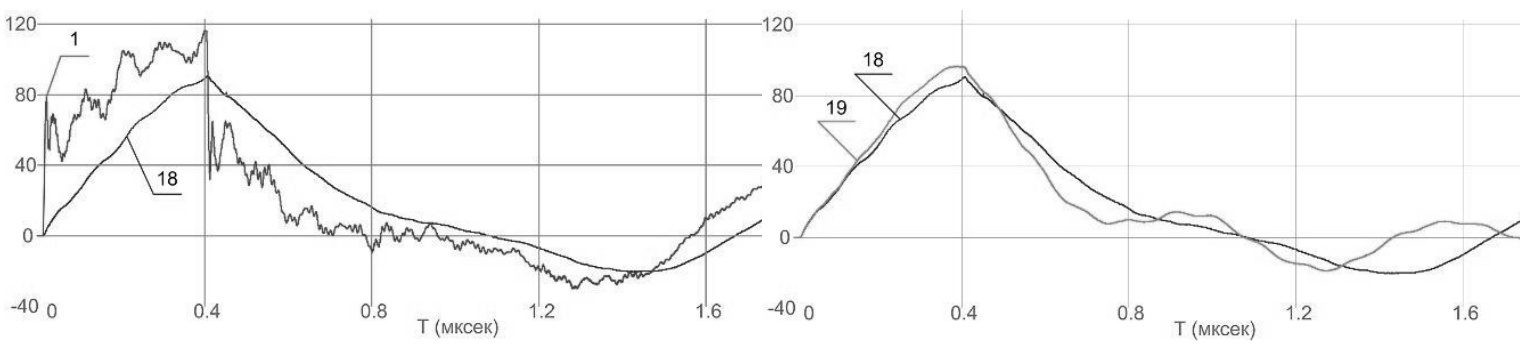

Figure 7. Combined impulses-responses on a free-defect scheme: curve 1 - front duration is $25 \mathrm{~ns}$, curve $18-400$ ns

\section{Conclusions}

Analysis of defectograms forms, obtained by simulation transformer tests in the program Micro-Cap-8, proved that it is possible to determine various defects in transformer windings; the simulation results are very close to the results of experimental studies based on a transformer physical model. Moreover, when front duration of the probe pulse is increased a possibility to determine the defects decreases. However, in case when front duration of the probe pulse is up to $400 \mathrm{~ns}$ winding turns cannot be determined.

It can be concluded that the LVP method aimed at the increase in steepening the probe pulse with reduced duration leads to the increase in sensitivity of the diagnostic procedure compared with the "classical" LVP method when pulses of microsecond duration are used.

\section{REFERENCES}

1. A.Yu. Khrennikov, V.A. Peredelsky, A.A. Safonov, V.A. Yakimov Application of a low-voltage pulses method to diagnose the condition of power transformers// Energetic, № 9, 2005. - P. 11-14.

2. A.Yu. Khrennikov Experience in detecting residual deformations of power transformer windings//Energetic, № 7, 2003. - P. 23-25.

3. A.A. Gribanov, S.O. Khomutov Mathematical modeling of the diagnostic signal in the assessment of power transformers using a low-voltage pulse method. Polzunovskii Vestnik № 2, 2010. - P.232-238.

4. V.A. Lavrinovich, A.V. Lavrinovich, A.V. Mytnikov. Development of Advanced Control State Technology of Transformer and Electric Motor Windings Based on Pulsed Method. - International Journal on "Technical and Physical Problems of Engineering", December 2012, Issue 13, Volume 4, Number 4, pp. 149-153. 